\title{
Impact of Self-Measured Blood Pressure Monitoring on Hypertension Control
}

\author{
Joel C. Marrs ${ }^{1 *}$ and Emily Cohen ${ }^{2}$ \\ ${ }^{1}$ Visiting Clinical Associate Professor at the University of Colorado Anschutz Medical Campus in the School of Medicine, Department of Pediatrics, Child \\ Health Associate/Physician Assistant Program, Aurora, Colorado, USA \\ ${ }^{2}$ Community Impact Director, American Heart Association, Southwest region, Denver, Colorado, USA at the time of this research. She is now owner and \\ lead educator at Tovah Consulting, LLC, Denver, Colorado, USA
}

${ }^{\star}$ Corresponding author: Dr. Marrs, Mail Stop F543, 13001 E. 17th Place, Room E7019, Aurora, Colorado, 80045, USA; Tel: 303-570-6014; Fax: 303-724-1350; E-mail: Joel.Marrs@cuanschutz.edu

Received: July 09, 2021; Accepted: July 15, 2021; Published: July 21, 2021

\begin{abstract}
Background: The American Heart Association developed the Check, Change, Control self-measured blood pressure program. The Check, Change, Control program (2016-2017 cohort) demonstrated higher odds of blood pressure control with checking self-measured blood pressure more frequently. The purpose of this study was to evaluate the impact of patient factors related to utilization of self-measured blood pressure and their association with blood pressure control in the 2017-2018 cohort.
\end{abstract}

Methods: Retrospective cohort study of adults enrolled in the Check, Change, Control program. The 2017-2018 cohort data was used to evaluate the hypertension population and their self-measured blood pressure values. The primary outcome measures differences between frequency of individual self-measured blood pressure reporting: self-measured blood pressure frequently $(>2$ times a month) and less frequent self-measured blood pressure $(<$ 2 times a month) and blood pressure control. Risk ratios for factors were calculated to determine association with reported self-measured blood pressure and blood pressure control.

Results: Overall, $37.3 \%$ uploaded $>2$ blood pressure values per month and $66 \%$ were female. The unadjusted risk ratios for having blood pressure $<$ $140 / 90 \mathrm{~mm} \mathrm{Hg}$ was higher for age > 60 vs. $<60$ years $(1.07 ; 95 \%$ Confidence Interval [CI] 1.04-1.10), lower for Black vs. nonblack adults (0.73; 95\% CI 0.67$0.79)$, higher for females vs. males (1.04; $95 \%$ CI 1.01-1.07), and lower for individual vs. employer enrollment (0.60; $95 \%$ CI 0.58-0.63).

Conclusion: One-third of adults reported self-measured blood pressure values greater than 2 times per month. Lower rates of blood pressure control (< $140 / 90 \mathrm{~mm} \mathrm{Hg}$ ) were associated with age $\geq 60$ years, Black race, male sex, and individual enrollment. An emphasis should be placed on the use of selfmeasured blood pressure to increase blood pressure control rates.

Keywords: Blood pressure measurement/monitoring, Health disparities, Health service research, Hypertension, Self-management

\section{Introduction}

Eighty-five million adults in the US have hypertension (HTN) and nearly half $(46.0 \%)$ of them have uncontrolled blood pressure (BP) despite medication and dietary interventions [1]. In addition, there are known racial disparities in BP control in Black compared to Non-Black adults in the US and across the world. Over the last 20 years, the use of self-measured blood pressure (SMBP) and/or home blood pressure monitoring (HBPM) has increased as a means to assist in the diagnosis and management of HTN. The 2017 ACC/AHA HTN guidelines endorse a class 1 level of evidence A recommendation for out-of-office BP measurement to confirm the diagnosis of HTN and for BP medication titration with telehealth counseling or clinical interventions [2-6]. The American Heart Association (AHA) recommends SMBP for evaluation of most patients with known or suspected HTN to assess response to treatment and possibly improve adherence [7]. The AHA has developed the Check, Change, Control (CCC) program, an evidence based HTN management program that incorporates concepts of remote monitoring
[8]. This program is designed to provide resources for individuals to be educated on and improve their lifestyle in order to improve their BP. In addition, the program allows for individuals to input their BP values in the CCC online program so they can track their $\mathrm{BP}$ as well this allows for them to communicate this information to their healthcare provider(s) if they so choose. There has been a previous analysis of the CCC program using the 2016-2017 cohort that demonstrated a higher odds of BP control for those program participants who checked their SMBP more frequently ( $>2$ times a month). The more recent cohort of data (2017-2018) has not been evaluated relative to the frequency of SMBP monitoring and HTN control.

\section{Aim of the Study}

Our study was designed to determine the prevalence of selfmeasured blood pressure (SMBP) by demographic characteristics and hypertension categories (BP controlled vs. uncontrolled) using the AHA CC Program (2017-2018) data set. 


\section{Methods and Materials}

A retrospective cohort study of men and women who have enrolled in the AHA CCC program from July 1, 2017 through June 30, 2018 was performed. During the study period, 18,617 adult individuals age 18 to 80 years of age who enrolled in the AHA CCC program uploaded 189,151 BP measurements. The cohort was reduced to those with at least 2 BP uploads, which narrowed the cohort to 10,638 individuals. The study population included individuals from across the United States who enrolled through their employer or self-enrolled. Those who uploaded values may or may not have elevated BP or HTN prior to enrollment. The data collection portal does not include any assessment of whether individuals have a diagnosis of HTN. The primary outcome measures differences between frequency of individual SMBP reporting: SMBP frequently ( $>2$ times a month) and less frequent SMBP $(<2$ times a month) and BP control. Risk ratios (RR) for self-reported demographic parameters included gender, age, race/ethnicity, systolic blood pressure (SBP), diastolic blood pressure (DBP), and enrolled in CCC through employer or as individual were calculated to determine association with reported SMBP and BP control.

Demographic parameters were defined as follows: gender (male or female); age (18-39 years, $40-59$ years, $\geq 60$ years); race/ethnicity categorized into 4 groups (Hispanic or Latino, Black, White, other); employer enrollment (yes or no) by enrollment code.

\section{Statistical Analysis}

Data analyses were conducted using SAS version 9.4 (SAS Institute, Cary, NC). Differences between demographic categorical variables were evaluated with the chi-square test for frequent SMBP and nonfrequent SMBP reporting. Differences between continuous variables (e.g. SBP, DBP) was evaluated with the t-test for frequent SMBP and nonfrequent SMBP reporting groups relative to BP control. An alpha level of 0.05 was used set to determine statistical significance. Statistically significant variables in the univariate analysis were adjusted for with adjusted risk ratios (RR) and 95\% confidence intervals (CI) calculated used a binomial regression model.

\section{Results}

Thirty-seven percent of program participants reported SMBP values greater than 2 times monthly during 2017-2018. Full patient demographics are reported out in Table 1. Program participants who uploaded SMBP values less frequently were more likely to be female, younger (18-39 years), and Black compared to other race/ ethnicities. Individuals enrolling with their employer were less likely to report SMBP frequently compared to those enrolling individually. No difference in BP control rates between frequent and less frequent SMBP monitoring was noted when looking at BP values $<140 / 90 \mathrm{~mm}$ $\mathrm{Hg}$ or $<130 / 80 \mathrm{~mm} \mathrm{Hg}$ in the univariate analysis. The mean SBP and DBP across SMBP reporting groups was $120 / 80 \mathrm{~mm} \mathrm{Hg}$.

The unadjusted RR for having BP $<140 / 90 \mathrm{~mm} \mathrm{Hg}$ was higher for age $>60$ vs. $<60$ years $(1.07 ; 95 \%$ CI 1.04-1.10), lower for Black vs. Non-Black adults $(0.73$; 95\% CI 0.67-0.79), higher for females vs. males (1.04; 95\% CI 1.01-1.07), and lower for individual vs. employer enrollment (0.60; 95\% CI 0.58-0.63). Complete unadjusted RR and adjusted RR are reported in Figure 1. After adjusting for SMBP frequency, age, race, gender, and type of enrollment, the RR for having

Table 1: Patient Demographics.

\begin{tabular}{|c|c|c|c|c|}
\hline Characteristic & $\mathbf{N}$ & Less Frequent SMBP (\%) & More Frequent SMBP (\%) & P-value \\
\hline Total & 10638 & $6668(62.7)$ & $3970(37.3)$ & \\
\hline Gender & 10577 & & & \\
\hline Female & 6939 & $4565(65.8)$ & $2374(34.2)$ & \multirow{2}{*}{$<0.0001$} \\
\hline Male & 3638 & $2055(56.5)$ & $1583(43.5)$ & \\
\hline Age Groups (years) & 10597 & & & \\
\hline $18-39$ & 2155 & $1576(23.8)$ & $579(14.6)$ & \multirow{3}{*}{$<0.0001$} \\
\hline $40-59$ & 5197 & $3198(48.2)$ & $1999(50.4)$ & \\
\hline$\geq 60$ & 3245 & $1856(28.0)$ & $1389(35.0)$ & \\
\hline Race/Ethnicity & 10638 & & & \\
\hline Hispanicor Latino & 947 & $618(9.3)$ & $329(8.3)$ & \multirow{4}{*}{$<0.0001$} \\
\hline White & 6762 & $4111(61.7)$ & $2651(66.8)$ & \\
\hline Black & 1878 & $1248(18.7)$ & $630(15.9)$ & \\
\hline Other & 1051 & $691(10.4)$ & $360(9.1)$ & \\
\hline Enrollment Group & 10638 & & & \\
\hline Individual & 4206 & $2073(31.1)$ & $2133(53.7)$ & \multirow{2}{*}{$<0.0001$} \\
\hline Employer & 6432 & 4595 (68.9) & $1583(46.3)$ & \\
\hline Mean ( \pm sd) SBP (mmHg) & 10638 & $127.8 \pm 17.7$ & $128.4 \pm 16.8$ & 0.0879 \\
\hline Mean $( \pm \mathrm{sd})$ DBP $(\mathrm{mmHg})$ & 10638 & $79.9 \pm 11.5$ & $79.9 \pm 11.2$ & 0.9025 \\
\hline $\mathrm{BP}<140 / 90 \mathrm{mmHg}$ & 10638 & $4718(70.8)$ & $2795(70.4)$ & 0.6992 \\
\hline $\mathrm{BP}<130 / 80 \mathrm{mmHg}$ & 10638 & $2633(39.5)$ & $1539(38.8)$ & 0.4611 \\
\hline
\end{tabular}




\begin{tabular}{|c|c|c|c|c|c|c|}
\hline Characteristic & $\mathbf{R R}$ & $95 \% \mathrm{Cl}$ & P-value & Adj. RR * & $95 \% \mathrm{Cl}$ & P-value \\
\hline $\begin{array}{l}\text { Age }(<60 \text { years vs } \geq 60 \\
\text { years) }\end{array}$ & 1.070 & 1.041-1.099 & $<0.0001$ & 1.051 & 1.023-1.079 & 0.0003 \\
\hline Race (Black vs nonblack) & 0.729 & $0.671-0.794$ & $<0.0001$ & 0.828 & $0.799-0.858$ & $<0.0001$ \\
\hline Gender (Females vs Males) & 1.040 & $1.008-1.072$ & 0.0130 & 1.040 & $1.012-1.062$ & 0.0030 \\
\hline $\begin{array}{l}\text { Enrollment (Individual vs } \\
\text { Employer) }\end{array}$ & 0.601 & $0.575-0.629$ & $<0.0001$ & 0.731 & $0.711-0.752$ & $<0.0001$ \\
\hline $\begin{array}{l}\text { BP less than } 140 / 90 \mathrm{~mm} \mathrm{Hg} \\
\text { (Less frequent vs More } \\
\text { frequent SMBP) }\end{array}$ & 1.006 & $0.974-1.039$ & 0.6992 & 0.951 & $0.930-0.973$ & $<0.0001$ \\
\hline $\begin{array}{l}\text { BP less than } 130 / 80 \mathrm{~mm} \mathrm{Hg} \\
\text { (Less frequent vs More } \\
\text { frequent SMBP) }\end{array}$ & 1.011 & $0.982-1.042$ & 0.4611 & 0.918 & $0.875-0.963$ & 0.0005 \\
\hline
\end{tabular}

Figure 1: Univariate and Multivariate Analysis of Hypertension Control Relative to Covariates and Frequency of SMBP.

$\mathrm{BP}<140 / 90 \mathrm{~mm} \mathrm{Hg}$ was higher for age $>60$ vs. $<60$ years $(1.05 ; 95 \%$ CI 1.02-1.08), lower for Black vs. Non-Black adults (0.83; $95 \%$ CI 0.80$0.86)$, higher for females vs. males (1.04; $95 \%$ CI 1.01-1.06), and lower for individual vs. employer enrollment (0.73; 95\% CI 0.71-0.75). In the adjusted model, having $\mathrm{BP}<140 / 90 \mathrm{~mm} \mathrm{Hg}$ and $<130 / 80 \mathrm{~mm} \mathrm{Hg}$ was lower for less frequent versus more frequent SMBP reporting $(0.95$; 95\% CI 0.93-0.97 and (0.92; 95\% CI 0.88-0.96, respectively).

\section{Discussion}

Study findings demonstrate that a small percentage of program participants (37\%) were able to report SMBP values greater than two times per month. Based on these numbers it appears SMBP monitoring is a viable option to assess out of office BP measurements and reporting to their healthcare providers. Additionally, these numbers highlight an educational opportunity to highlight the importance of SBMP and the role it plays in the prevention and management of patients with hypertension. This approach of SBMP monitoring is supported by the 2017 ACC/AHA HTN guideline recommendations as a useful screening and monitoring tool for those with and without HTN.

This analysis demonstrated lower rates of BP control $(<140 / 90$ $\mathrm{mm} \mathrm{Hg}$ ) associated with age $\geq 60$ years, Black race, male gender, and individual enrollment in the unadjusted and adjusted models. These associated lower rates of BP control align with previous data demonstrating that age, race, and gender contribute to variance in $\mathrm{BP}$ control rate across populations. Further, when adjusting for age, race, gender, and type of enrollment, lower rates of BP control at $<140 / 90$ $\mathrm{mm} \mathrm{Hg}$ and $<130 / 80 \mathrm{~mm} \mathrm{Hg}$ were seen in the less frequent versus more frequent SMBP reporting groups. Based on these findings, further focus needs to be placed on populations demonstrating lower BP control rates.

\section{Study Limitations}

One limitation of our study is the retrospective nature of the study which depends on the accuracy of data input into the AHA CCC online platform by participants in the program. Secondly, the retrospective study design could only demonstrate associations with and not any causal relationships with SBMP and BP control rates.

\section{Conclusions}

One-third of adults reported SMBP values greater than 2 times per month. Lower rates of BP control $(<140 / 90 \mathrm{~mm} \mathrm{Hg})$ were associated with age $\geq 60$ years, Black race, male sex, and individual enrollment. Overall, the use of SMBP should be recommended to help individuals to improve and maintain BP control and to identify factors associated with lower BP control rates.

\section{References}

1. Virani AA, Alonso A, Aparicio HJ, et al. (2021) American Heart Association Statistics Committee and Stroke Statistics Subcommittee. Heart disease and stroke statistics-2021 update: a report from the American Heart Association. Circulation 143: e254-743.

2. Whelton PK, Carey RM, Aronow WS, et al. (2018) 2017 ACC/AHA/AAPA/ABC/ ACPM/AGS/APhA/ASH/ASPC/NMA/PCNA Guideline for the Prevention, Detection, Evaluation, and Management of High Blood Pressure in Adults: A Report of the American College of Cardiology/American Heart Association Task Force on Clinical Practice Guidelines. J Am Coll Cardiol 71: e127-e248. [crossref]

3. Uhlig K, Balk EM, Patel K, et al. (2012) Self-Measured Blood Pressure Monitoring: Comparative Effectiveness. Rockville, MD: Agency for Healthcare Research and Quality (U.S.)

4. Margolis KL, Asche SE, Bergdall AR, et al. (2013) Effect of home blood pressure telemonitoring and pharmacist management on blood pressure control: a cluster randomized clinical trial. JAMA 310: 46-56. [crossref]

5. McManus RJ, Mant J, Haque MS, et al. (2014) Effect of self-monitoring and medication self-titration on systolic blood pressure in hypertensive patients at high risk of cardiovascular disease: the TASMIN-SR randomized clinical trial. JAMA 312: 799-808. [crossref ]

6. Siu AL (2015) Screening for high blood pressure in adults: U.S. Preventive Services Task Force recommendation statement. Ann Intern Med 163: 778-86. [crossref]

7. Muntner P, Shimbo D, Carey RM, et al. (2019) Measurement of blood pressure in humans: a scientific statement from the American Heart Association. Hypertension 73: e35-e66. [crossref ]

8. Thomas KL, Shah BR, Elliot-Bynum S, et al. (2014) Check it, change it a communitybased, multifaceted intervention to improve blood pressure control. Circ Cardiovasc Qual Outcomes 7: 828-834. [crossref]

\section{Citation:}

Marrs JC, Cohen E (2021) Impact of Self-Measured Blood Pressure Monitoring on Hypertension Control. J Cardiol Clin Pract Volume 4(2): 1-3. 